\title{
Remarkable electron and phonon transports in low-cost SnS: A new promising thermoelectric material
}

\author{
Wenke $\mathrm{He}^{1,2}$, Ran $\mathrm{Ang}^{1}$ and Li-Dong Zhao ${ }^{2 *}$
}

\begin{abstract}
As a deceptively simple material, SnS has received extensive attention in the thermoelectric community recently due to its fascinating electron and phonon transport features, making it a very promising thermoelectric candidate. In this review, we first discuss the fundamental characteristics of SnS, including crystal structures, electronic and phononic band structures, and physical or chemical properties. Meanwhile, the approaches of improving thermoelectric performance are summarized, including simultaneous carrier concentration tuning and carrier mobility improvement, multiple valence bands transport and their synergetic optimization, and anharmonicity and phonon "softening" behavior. We also compare the difference in electrical and thermal transport properties between crystals and polycrystalline SnS. Then, theoretical calculations to predict the maximum $Z T$ in SnS system are also established for potential performance enhancement. Finally, some future possible strategies are proposed to aim at further promoting the figure of merit of SnS. The exploration and research on this new emerged material can contribute the thermoelectrics toward practical applications to meet market demands of low-cost, higheffectiveness, and environmental compatibility.
\end{abstract}

Keywords: SnS, thermoelectrics, electron and phonon, low-cost, ZT

\section{INTRODUCTION}

Since exploring new energy sources or improving energy utilization patterns has become a long-term effective means to cope with the ever-increasing demands for energy, we have witnessed the prosperity of energy technology in many areas over the last few decades. Thermoelectric technology, particularly, as a renewable energy utilization technology has sprung up in recent years, which allows the direct conversion to electricity from harvesting wasted heat $[1,2]$. For a given material, its thermoelectric conversion efficiency depends on a dimensionless figure of merit $Z T$. It is defined as $Z T=S^{2} \sigma T /\left(\kappa_{\text {lat }}+\kappa_{\text {ele }}\right)$, where $S, \sigma, T$, $\kappa_{\text {lat }}$ and $\kappa_{\text {ele }}$ are the Seebeck coefficient, electrical conductivity, absolute temperature in Kelvin, and thermal conductivity in lattice and electron, respectively [3,4]. Obviously, prominent thermoelectric materials should be qualified with large Seebeck coefficient, high electrical and low thermal conductivity simultaneously [5-8]. The difficulty in optimizing ZT, however, is the complex coupling relationship between these parameters, and thus making a balance between them to achieve an overall $Z T$ enhancement can yet be regarded as an effective way [9-11].

Under the tremendous efforts, increasing ideals for improving $Z T$ have emerged and considerable progress has been made, including the discovery of new material systems [12-15], establishment of innovative transport theories [16-21] and development of advanced fabrication processes as well as rational material design strategies [1,4,22-25]. On the other hand, the progress on thermoelectric devices is slow, and still dominated by conventional systems, such as $\mathrm{Bi}_{2} \mathrm{Te}_{3}$ [26-28] and $\mathrm{PbTe}$ [29-33]. However, in addition to containing rare heavy elements, these traditional materials have disadvantages of toxicity and high cost. As a result, these factors make it difficult to achieve high yield and economic benefits, which limits their application. Certainly, low thermoelectric conversion efficiency remains a major barrier to the device application. Therefore, developing low-cost, high-performance, environmentallyfriendly and lightweight thermoelectrics is of great significance to promote the practical application. Recently, in a series of studies, it is found that tin sulfide $(\mathrm{SnS})$ is a rising star in thermoelectric community as it possesses all the above virtues [34-37].

In the past, $\mathrm{SnS}$ was ignored in the thermoelectric field owing to its poor electrical transport caused by the large bandgap $(\sim 1.2 \mathrm{eV})$ [36-40]. Nevertheless, it is encouraging that this material was found to possess intrinsically low thermal conductivity and came into the sight of researchers. In recent years, continuous progress has been made in $\mathrm{SnS}$ and the $Z T$ values are also being broken through [34-37,39,41-51], as shown in Fig. 1a. Along with the thorough studies, the thermoelectric features of this material have also been exploited. Unlike conventional thermoelectric systems, SnS is a layered compound with lower symmetry in structure [38,52]. This asymmetric structure is the basis of excellent electronic and phononic band structures found in this material. For instance, Seebeck coefficient can be enhanced from the complex electronic band structure [34-36,53-55], electrical conductivity can be compensated by utilizing the high in-plane carrier mobility of the layered structure in its crystal form [34-36,50,56], and the ultralow thermal conductivity originates from the strong lattice anharmonicity thus suppressing phonon transport [34,39]. Besides, SnS consists of low-cost and non-toxic elements, with both environmental compatibility and abundant reserves (e.g.,

\footnotetext{
${ }^{1}$ Key Laboratory of Radiation Physics and Technology, Ministry of Education, Institute of Nuclear Science and Technology, Sichuan University, Chengdu 610064, China

${ }^{2}$ School of Materials Science and Engineering, Beihang University, Beijing 100191, China

* Corresponding author (email: zhaolidong@buaa.edu.cn)
} 

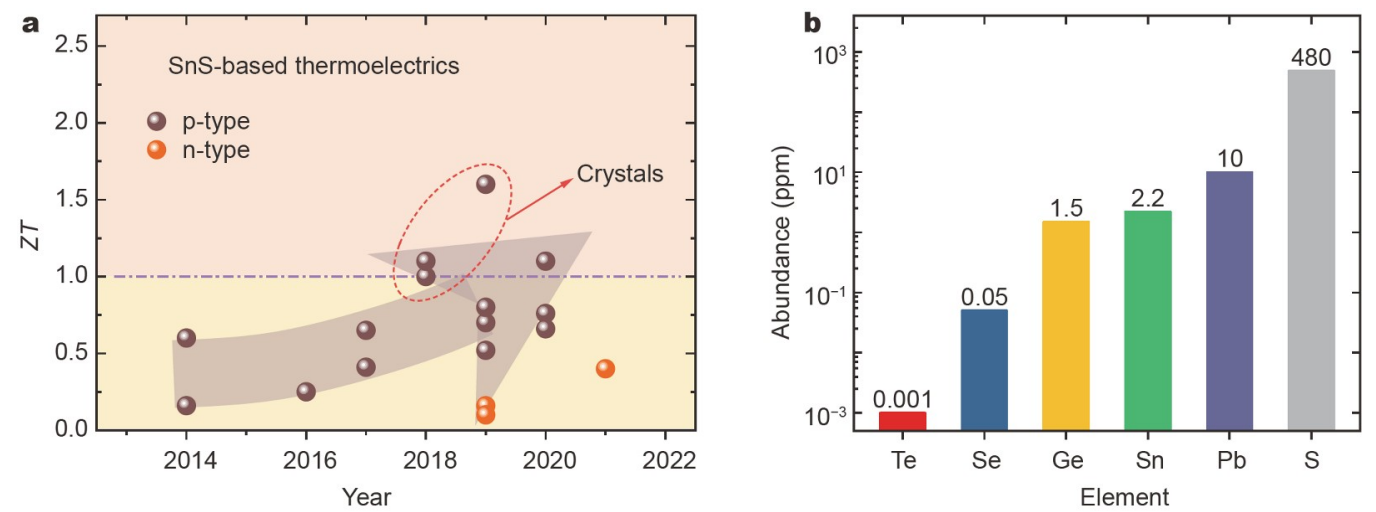

Figure 1 (a) ZT values of SnS-based thermoelectrics. (b) Earth abundance of IV-VI group elements.

Te $\sim 0.001 \mathrm{ppm}, \mathrm{Se} \sim 2.2 \mathrm{ppm}, \mathrm{S} \sim 480 \mathrm{ppm}$ ), making it superior to other IV-VI group semiconductors strikingly (Fig. 1b) $[57,58]$. Therefore, SnS-based materials become one of the thermoelectric hotspots, and growing research achievements have been reported. It is essential to review and make a summary for the latest progress made in this newcome material.

From this view, we summarize the electron and phonon transport behaviors in SnS and strategies for boosting its thermoelectric performance, including carrier concentration (Fermi level) tuning, carrier mobility improvement, and synergetic optimization between carrier effective mass and mobility through manipulating electronic band structure, etc. Firstly, the crystal structure is introduced, and the produced differences in electrical and thermal transports caused by the anisotropic structure are discussed. Then we highlight the electrical transport features for both $\mathrm{p}$ - and n-type $\mathrm{SnS}$, including the huge distinctions in electrical conductivity arising from the different crystal forms of the material, multiple valence band structure for Seebeck coefficient enhancement, and temperature-dependent synergetic transport characteristics of three separate electronic valence bands for high power factor $(P F)$. In addition, we also discuss the charge transports from the aspects of formation energy and atomic orbital contribution in n-type SnS. On the other hand, we put emphasis on the anharmonicity and phonon "softening" to the thermal conductivity. Finally, the relationship between maximum $Z T$ and quality factor $B$ is established and the potential performance optimization is predicted in both $\mathrm{p}$ - and n-type SnS-based materials.

\section{CRYSTAL STRUCTURE}

$\mathrm{SnS}$ is crystallized in orthorhombic structure and belongs to the space group Pnma (\#62) at room temperature. This kind of layered compound shows obvious anisotropy in the spatial structure, with $a=11.19 \AA$, $b=3.98 \AA$ and $c=4.33 \AA$ [34]. The atomic arrangements in SnS crystal structure along the three crystallographic directions are shown in Fig. 2a. Like the structure in SnSe [59-62], one $\mathrm{Sn}$ atom in $\mathrm{SnS}$ can be bonded with seven surrounding $\mathrm{S}$ atoms to form a highly distorted $\mathrm{SnS}_{7}$ coordination polyhedral, where five bonds (bonding length $d<$ $3.2 \AA)$ are formed within the two-atom-thick layer and two longer bonds $(d>3.5 \AA$ ) formed outside the layer. Because of the longer $\mathrm{Sn}-\mathrm{S}$ bonds, the interatomic bonding is weak and easy to cleave along the interlayer direction ( $a$-axis), as shown in Fig. $2 \mathrm{~b}$. Therefore, from the perspective of $a$-axis ( $b-c$ plane, in Fig. 2a) or the cleavage plane of crystal (in Fig. 2b), the layered structure is in favor of carrier migration, and thus higher carrier mobility in $\mathrm{SnS}$ along the in-plane direction. It can be seen that the twoatom-thick layers are made up of $\mathrm{Sn}$ and $\mathrm{S}$ atoms that are staggered up and down along the $a$-axis direction. Besides, along the $b$ or $c$-axis, atoms are arranged to form periodic spring-like structures or zigzag $\mathrm{Sn}-\mathrm{S}$ type chains. It is worth noting that the weaker bonding along the $c$-axis will lead to lower thermal conductivity than that along the $b$-axis. Certainly, the thermal conductivity along the $a$-axis will be much lower due to the longer and weaker bonding between the layers, thus impeding thermal transport greatly.

From the above discussion, the anisotropic structure in SnS will cause huge deviations in electrical or thermal transport along the three axial directions. Therefore, it is crucial to conducting electrical and thermal transport properties measurements along each direction separately. For SnS crystals, the samples can be cut along the three crystallographic axes, as shown in Fig. $2 \mathrm{~b}$. The $a$-axis is easy to be distinguished, while the $b$ - or $c$-axis can be identified by a conventional Laue X-ray diffraction (XRD) method [6,34]. On the other hand, although polycrystalline SnS samples are composed of numerous crystal grains, the grains will be arranged in a preferred orientation during the sintering or hot-pressing process due to the layered structure, thus resulting in a strong anisotropy $[37,46,50]$. Hence, it is also necessary to perform the thermoelectric measurements along both the two directions of sintering pressure $(/ / P$ and $\perp P$ ) separately, as shown in Fig. 2c.

\section{CARRIER CONCENTRATION OPTIMIZATION AND MOBILITY IMPROVEMENT}

Owing to the existence of intrinsic Sn vacancy, SnS samples synthesized under strict stoichiometric ratio still exhibit p-type conduction behavior $[38,63]$. However, the carrier concentration is very low due to its large band gap. As a result, the final ZT values are below $\sim 0.4$ with the carrier concentrations lower than $\sim 5 \times 10^{17} \mathrm{~cm}^{-3}$ in undoped SnS samples (Fig. 3a) [37,42,45-48]. Carrier concentration tuning is one regular but the most effective optimization strategy to improve ZT. Generally, heterovalent cation doping in $\mathrm{Sn}$ sites can elevate the carrier concentration, such as alkali-metal cations $\left(\mathrm{Li}^{+}\right.$and $\left.\mathrm{Na}^{+}\right)[34,48]$ or other monovalent cations $\left(\mathrm{Ag}^{+}\right.$and $\left.\mathrm{Tl}^{+}\right)[37,43]$. In addition, extra Sn vacancy can also achieve carrier concentration improvement. After effective doping, the optimal hole carrier concentrations range from $\sim 1 \times 10^{18}$ to $\sim 3 \times 10^{19} \mathrm{~cm}^{-3}$, as well as the $Z T$ values are promoted strikingly [34-37,39,44,45,47-49]. 

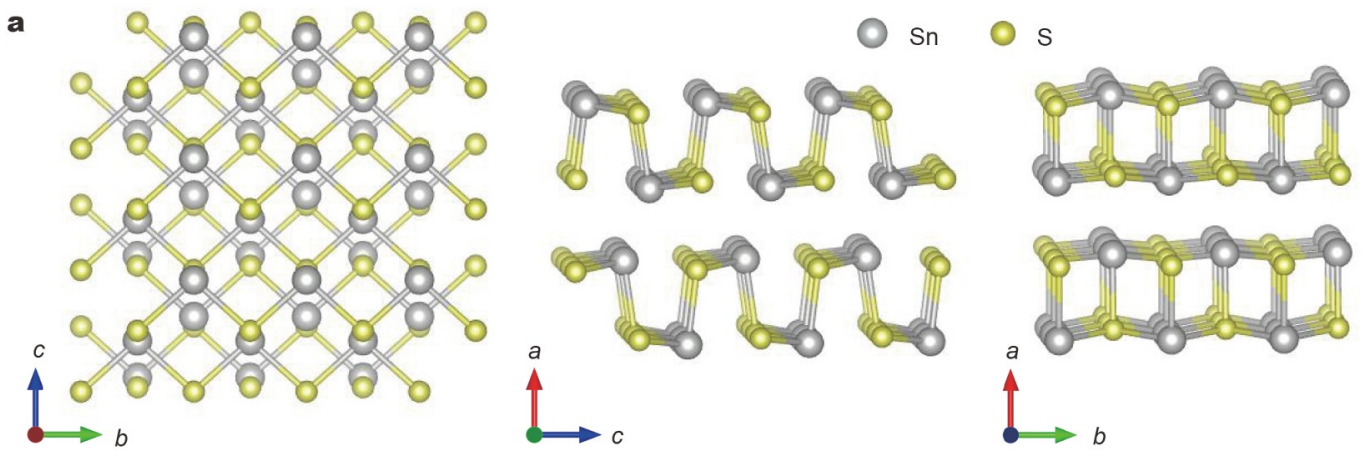

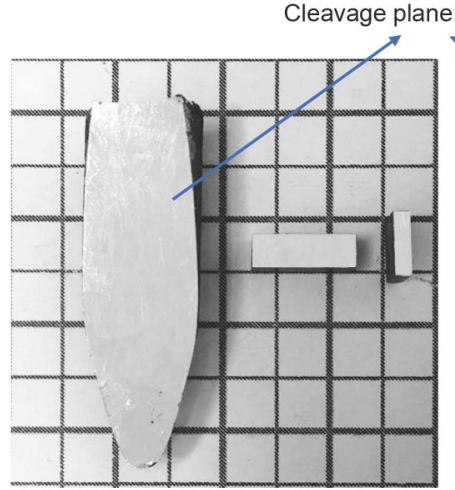

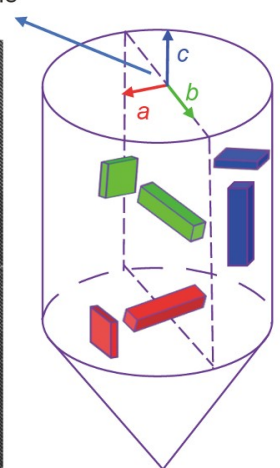

c

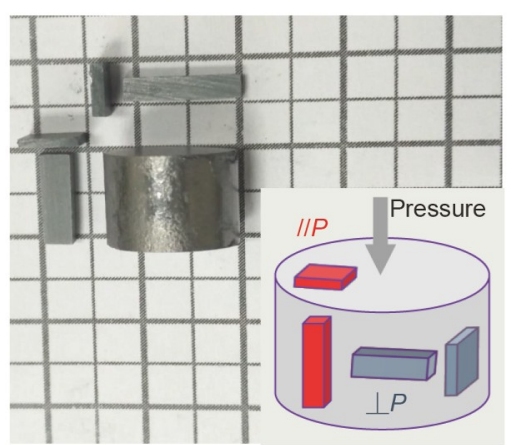

Figure 2 Crystal structures, typical crystalline and polycrystalline samples of SnS. (a) Crystal structures along the $a, b$ and $c$ axes, respectively. (b) A typical crystal cleaved along cleavage plane (100) and the corresponding diagram of cutting samples along the three crystallographic directions. Reproduced with permission from Ref. [36]. Copyright 2019, The American Association for the Advancement of Science. (c) Polycrystalline SnS sample and the corresponding diagram of cutting samples along $/ / P$ and $\perp P$, respectively.

Noticeably, the $Z T$ values exceed $\sim 1.0$ in Na-doped SnS crystals and even reach $\sim 1.6$ after further Se alloying [34-36]. Therefore, it is shown that the thermoelectric performance in SnS crystals is superior to the polycrystalline samples.

According to the Drude-Sommerfeld free electron model $\sigma=$ $n e \mu$ (where $\sigma$ is the electrical conductivity, $n$ is the carrier concentration, $e$ is the electronic charge and $\mu$ is the carrier mobility), electrical conductivity enhancement is related not only to the carrier concentration, but also to the carrier mobility. For the polycrystalline $\mathrm{SnS}$ samples, the electrical conductivity is as low as $\sim 10^{-3} \mathrm{~S} \mathrm{~cm}^{-1}$ and can only reach $\sim 10 \mathrm{~S} \mathrm{~cm}^{-1}$ after carrier concentration tuning at room temperature (Fig. 3b) [39,44,45]. Nonetheless, when SnS is prepared to crystals, the electrical conductivity can be further boosted from the improved carrier mobility due to the elimination of grain boundary. Therefore, the electrical conductivity in Na-doped $\mathrm{SnS}$ crystals can reach above $\sim 500 \mathrm{~S} \mathrm{~cm}^{-1}$ at room temperature, which is increased by one or two orders of magnitude $[34,35]$. It is interesting to probe the discrepancy of carrier mobility between different crystal forms in SnS. Within the whole temperature range, the carrier mobility in SnS crystals approximatively follows a relationship of $\sim T^{-3 / 2}$, indicating that the carriers are mainly scattered by acoustic phonon [64]. Being different from the change pattern of crystals, the carrier mobility decreases with temperature as $\sim T^{-3 / 2}$ after $\sim 450 \mathrm{~K}$, while the deviation occurs near room temperature in polycrystalline samples. The subdued carrier mobility at low temperatures is ascribed to the strong grain boundary potential barrier in polycrystalline SnS [65]. The grain boundary scattering has also been observed in other polycrystalline thermoelectric systems, such as SnSe [66-68], GeSe
[69], $\mathrm{Mg}_{3}(\mathrm{Sb}, \mathrm{Bi})_{2}$ [70]. Besides, compared with the crystals, the overall carrier mobility is still lower in polycrystalline SnS (apart from the suppressed mobility below $\sim 450 \mathrm{~K}$ ) in the entire working temperature range, indicating a constant difference in carrier mobility caused by grain boundary. On the other hand, this demonstrates that grain size can regulate the carrier mobility, namely, the larger grain size, the higher carrier mobility. Thereby, increasing the grain size by means of heat treatment process could be a simple and effective approach to enhance the carrier mobility in polycrystalline $\mathrm{SnS}$. This method has been applied in polycrystalline $\mathrm{Mg}_{3} \mathrm{Sb}_{1.5} \mathrm{Bi}_{0.5}$ samples by annealing, which results in a decline in grain boundary scattering as the grain size increases [70]. Similarly, the deleterious effect of carrier mobility caused by grain boundary can be eliminated completely in SnS crystals with single large grain size. For undoped SnS crystals, the carrier concentration is $\sim 10^{17} \mathrm{~cm}^{-3}$ level at room temperature and remains unchanged before $\sim 600 \mathrm{~K}$, which mainly comes from the ionization of impurities induced by the intrinsic defects ( $\mathrm{Sn}$ vacancy). After that, the carrier concentration has an exponential increment triggered by the thermal activation with rising temperature, resulting in improved electrical conductivity (inset of Fig. 3d). It should be noted that the carrier concentration achieves an increase of two orders of magnitude after $\mathrm{Na}$ doping $\left(\sim 10^{19} \mathrm{~cm}^{-3}\right)$, and is almost constant as temperature rises, thus showing a continuous decline in electrical conductivity but still higher than the undoped one (inset of Fig. 3d). In summary, hole-doped SnS crystals demonstrate excellent electrical conductivity, which benefits from the enhancement of both carrier concentration and carrier mobility. 

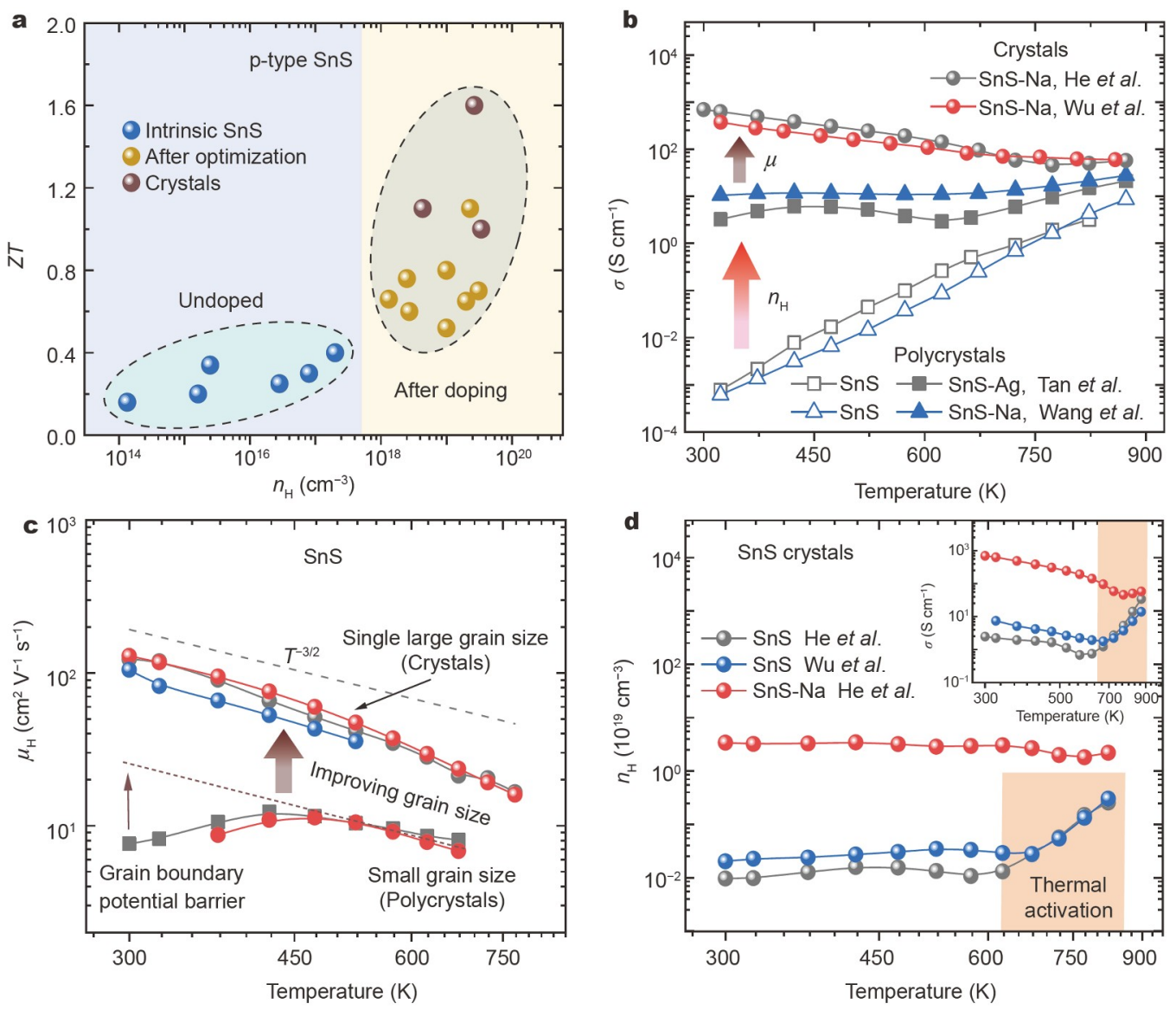

Figure $3 Z T$ values and electrical transport properties as a function of temperature for both $\mathrm{SnS}$ crystals and polycrystalline samples. (a) $Z T$ values under different carrier concentrations for p-type SnS. (b) Electrical conductivity. (c) Carrier mobility. (d) Carrier concentration.

\section{Multiple valence band structure}

Since the expression of $Z T$ is proportional to the square of Seebeck coefficient $\left(S^{2}\right)$, the enhancement in $S$ seems more effective than that in electrical conductivity for improving $Z T$ s. For a degenerated semiconductor with parabolic band dispersion, the Seebeck coefficient is approximately expressed by Equation (1) [71]:

$S=\frac{8 \pi^{2} k_{\mathrm{B}}{ }^{2}}{3 e h^{2}} m_{\mathrm{d}}^{*} T\left(\frac{\pi}{3 n}\right)^{2 / 3}$.

where $k_{\mathrm{B}}$ and $h$ are the Boltzmann constant and Plank constant, respectively. Obviously, to obtain a large Seebeck coefficient at a given carrier concentration, a high density of states (DOS) effective mass $\left(m_{\mathrm{d}}{ }^{*}\right)$ is required. In addition, according to the formula of $m_{\mathrm{d}}{ }^{*}=N_{\mathrm{v}}{ }^{2 / 3} \mathrm{~m}_{\mathrm{b}}{ }^{*}$, a large $m_{\mathrm{d}}{ }^{*}$ can be realized by increasing either the band degeneracy number $\left(N_{\mathrm{v}}\right)[18,72-74]$ or the single-band effective mass $\left(m_{\mathrm{b}}{ }^{*}\right)[75,76]$. There are two situations in increasing $N_{\mathrm{v}}$ : one is achieving high valley degeneracy via changing the symmetry of crystals (valley degeneracy) [77], and the other is enabling the band extrema of bands as many as possible within the same or similar Fermi energy level (orbital degeneracy) [60,78].

As mentioned above, $\mathrm{SnS}$ is crystallized in a layered structure with strong anisotropy, and this asymmetric structure generally splits the electronic band due to the lower symmetry [79]. Thus, the electronic transport can directly enhance the Seebeck coefficient if many split bands have comparable energy and converge together to result in an increased valley degeneracy (orbital degeneracy). As shown in Fig. 4a, the density functional theory (DFT) calculation reveals a multiple electronic valence band structure in SnS. The first valence band maximum (VBM1) lies in $\Gamma-Z$ direction while the second valence band maximum (VBM2) locates on $U$ point, and the energy difference between the first two valence bands $\left(\Delta E_{12}\right)$ is $\sim 0.056 \mathrm{eV}$. The third VBM lies below the VBM1 in the same direction with the energy difference $\Delta E_{13}=0.065 \mathrm{eV}$, whereas the fourth VBM lies in the $\Gamma-Y$ direction with $\Delta E_{14}=0.118 \mathrm{eV}$ [34]. It is worth nothing that, the energy gap between the first and forth bands $\left(\Delta E_{14}\right)$ is even smaller than that of the $\Delta E_{13}=0.13 \mathrm{eV}$ in SnSe [72]. The DFT calculations demonstrate a very small energy difference between these bands, and thus the increment in $N_{\mathrm{v}}$ can be realized once the Fermi level was pushed down into deeper level with increasing carrier concentration. Therefore, within the small energy region, the increased number of valleys results in an enhancement in effective mass.

Experimentally, the angle-resolved photoemission spectroscopy (ARPES) measurements confirmed the multiple valence bands and corresponding locations in SnS [34]. The $b-c$ plane corresponds to the $Y-\Gamma-Z$ plane in the Brillouin zone, where three valence bands are located at binding energies below $\sim 0.4 \mathrm{eV}$ along different $k$-space directions, as shown in Fig. $4 \mathrm{~b}$. Concretely, one VBM (marked as VBM2) locates along the $\Gamma-Y$ direction and two VBMs (marked as VBM1 and 1') situate along the $\Gamma-Z$ direction, corresponding to the DFT calculation results (Fig. 4c). From Fig. 4b, it is hard to distinguish the VBMs1 and $l^{\prime}$ in the $k-E$ map, indicating a small energy offset and close 

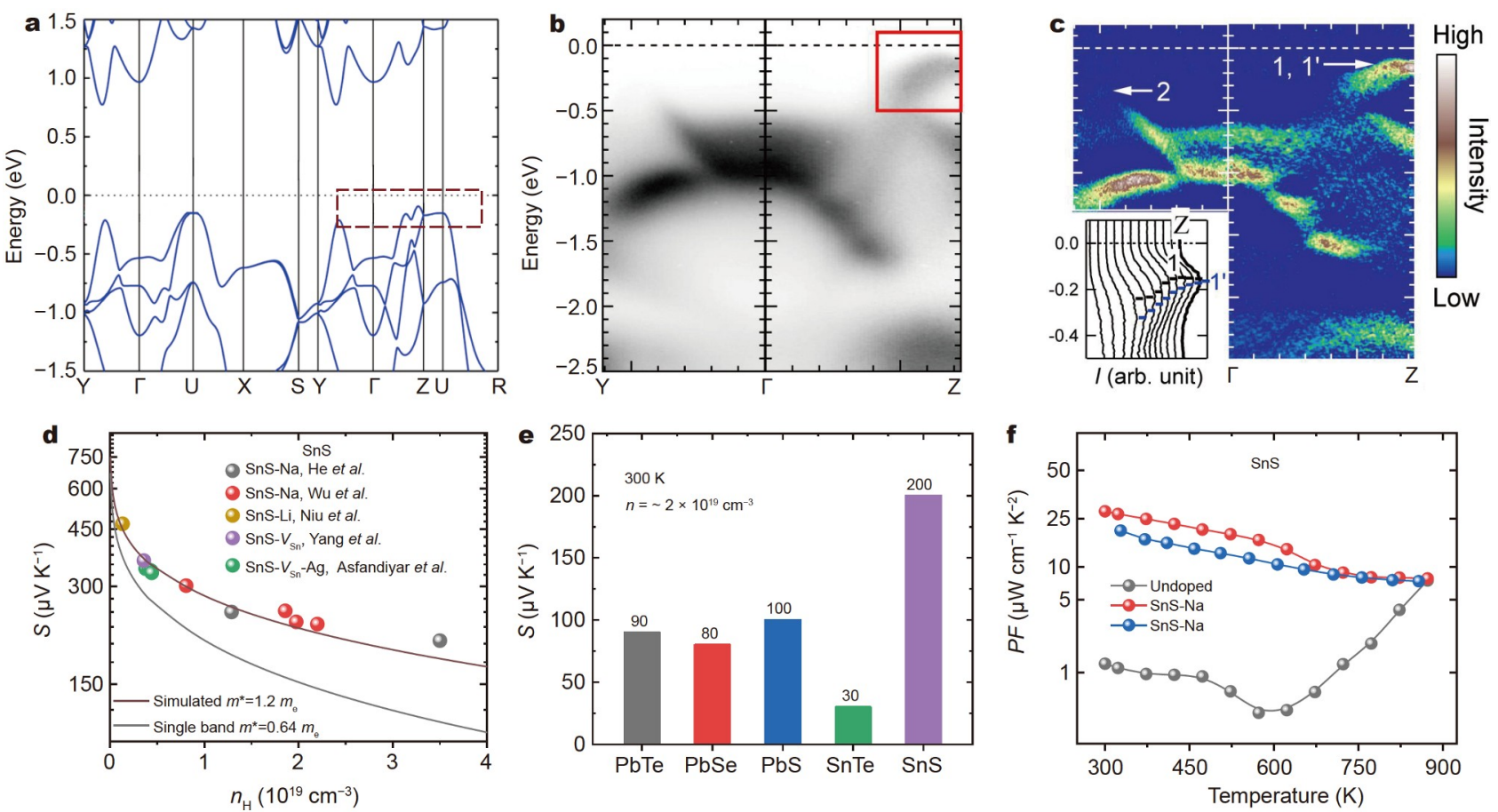

Figure 4 Electronic band structures, Seebeck coefficient and $P F$ for SnS. (a) Electronic valence band structure (the red box includes four valence band maximum points). (b) ARPES band dispersion along the $Y-\Gamma-Z$ direction. (c) Second derivative of (b), and 1, 1' and 2 points to the VBMs. Inset: energy distribution curves (EDCs) near VBMs 1 and 1' (red box in (b)). Reproduced with permission from Ref. [34]. Copyright 2018, the Royal Society of Chemistry. (d) Pisarenko line of Seebeck coefficient as a function of carrier concentration at room temperature. (e) Seebeck coefficients for group IV-VI thermoelectrics at a carrier concentration of $\sim 2 \times 10^{19} \mathrm{~cm}^{-3}$. (f) PFs for undoped and Na-doped SnS crystals.

position. However, their accurate $k$ locations and binding energies can be derived through the detailed peak fitting of the ARPES energy distribution curves (EDCs), as shown in the left bottom of Fig. 4c. Therefore, the coordinates of three VBMs in the $Y-\Gamma-Z$ plane are $(0,0.64,-0.148)$ in VBM1, $(0,0.725$, $-0.164)$ in VBM1' $(Z$ point) and $(0.48,0,-0.341)$ in VBM2, respectively, where $\left(k_{y}, k_{z}, E\right)$ are in units of $\AA^{-1}, \AA^{-1}$ and $\mathrm{eV}$. The energy difference within the three VBMs is $\sim 0.193 \mathrm{eV}$, comparable to the DFT calculations. The well supported ARPES results and DFT calculations demonstrate that SnS possess a multiple valence band structure.

The Pisarenko function can estimate the effective mass based on the relationship between Seebeck coefficient and carrier concentration $[1,22]$. According to the DFT calculation, the first valence band effective mass in $\mathrm{SnS}$ is $0.64 \mathrm{~m}_{\mathrm{e}}$, and the plotted Pisarenko line is shown in Fig. 4d. Herein, some reported data of SnS samples are provided within a carrier concentration range $\left(\sim 1.5 \times 10^{18}\right.$ to $\left.\sim 3.5 \times 10^{19} \mathrm{~cm}^{-3}\right)[34,35,46-48]$. It is obvious that the Seebeck coefficients of all samples are above the single-band Pisarenko curve, implying an increased effective mass. If these data are fitted by a Pisarenko plot, a simulated effective mass $\left(m_{\mathrm{d}}{ }^{*}\right) \sim 1.2 m_{\mathrm{e}}$ can be yield, which indicates that multiple valence bands are involved and contribute to $m_{\mathrm{d}}{ }^{*}$ enhancement. However, with increasing carrier concentration $\left(n_{\mathrm{H}}>\right.$ $\sim 2 \times 10^{19} \mathrm{~cm}^{-3}$ ), the deviation appears, manifesting the additional valence bands can be further activated. It is interesting to compare the large Seebeck coefficient in SnS with those of other IV-VI group materials with high symmetric rock-salt structures. As shown in Fig. 4e, under the carrier concentration of $\sim 2 \times 10^{19} \mathrm{~cm}^{-3}$ at $300 \mathrm{~K}$, the Seebeck coefficient in $\mathrm{SnS}$ is $\sim 200 \mu \mathrm{V} \mathrm{K} \mathrm{K}^{-1}$, higher than that in PbTe $\left(\sim 90 \mu \mathrm{V} \mathrm{K}^{-1}\right)[80], \mathrm{PbSe}$ $\left(\sim 80 \mu \mathrm{V} \mathrm{K}^{-1}\right) \quad$ [81], PbS $\quad\left(\sim 100 \mu \mathrm{V} \mathrm{K}^{-1}\right) \quad$ [82] and SnTe $\left(\sim 30 \mu \mathrm{V} \mathrm{K}^{-1}\right)$ [83]. This indicates that the low symmetric structure in $\mathrm{SnS}$ is conducive to the complex multiple electronic band structure. Moreover, the multiple bands maintain the high Seebeck coefficient and ensure the electrical conductivity improvement as the carrier concentration is increased, getting rid of the strong inverse relationship between Seebeck coefficient and carrier concentration in a single band. That is to say, the net effect of increasing band degeneracy $N_{\mathrm{v}}$ via improving $n$ will bring about an increase in electrical transport property. As a result, a high $P F$ of $\sim 30 \mu \mathrm{W} \mathrm{cm}{ }^{-1} \mathrm{~K}^{-2}$ at $300 \mathrm{~K}$ and an overall electrical performance improvement in $\mathrm{SnS}$ can be achieved once the carrier concentration is increased (Fig. 4f).

\section{Synergetic optimization between multiple bands}

The complex band structure in SnS facilitates the simultaneous improvement in both Seebeck coefficient and electrical conductivity after carrier optimization. Apart from this case, the electronic band structure tailoring is another powerful strategy for boosting electrical transport properties. For a single parabolic band (SPB) structure, the carrier effective mass is inversely proportional to the carrier mobility as follows: $\mu=e \tau / \mathrm{m}^{*}$. Generally, a high carrier mobility serves to promote the electrical conductivity $(\sigma=n e \mu)$ but unfortunately results in a very low $m^{*}$, thus producing a small Seebeck coefficient [84]. However, for a multiple band structure like $\mathrm{SnS}$, the large DOS effective mass mainly comes from the increased $N_{\mathrm{v}}$ rather than the single band $m_{\mathrm{b}}{ }^{*}$. Therefore, making a compromise between $\mu$ and $m_{\mathrm{b}}{ }^{*}$ is the key for further improvement in SnS. Alloying with a specific element is an effective way to not only shape the band structure but adjust the difference between multiple bands [36]. After Se 

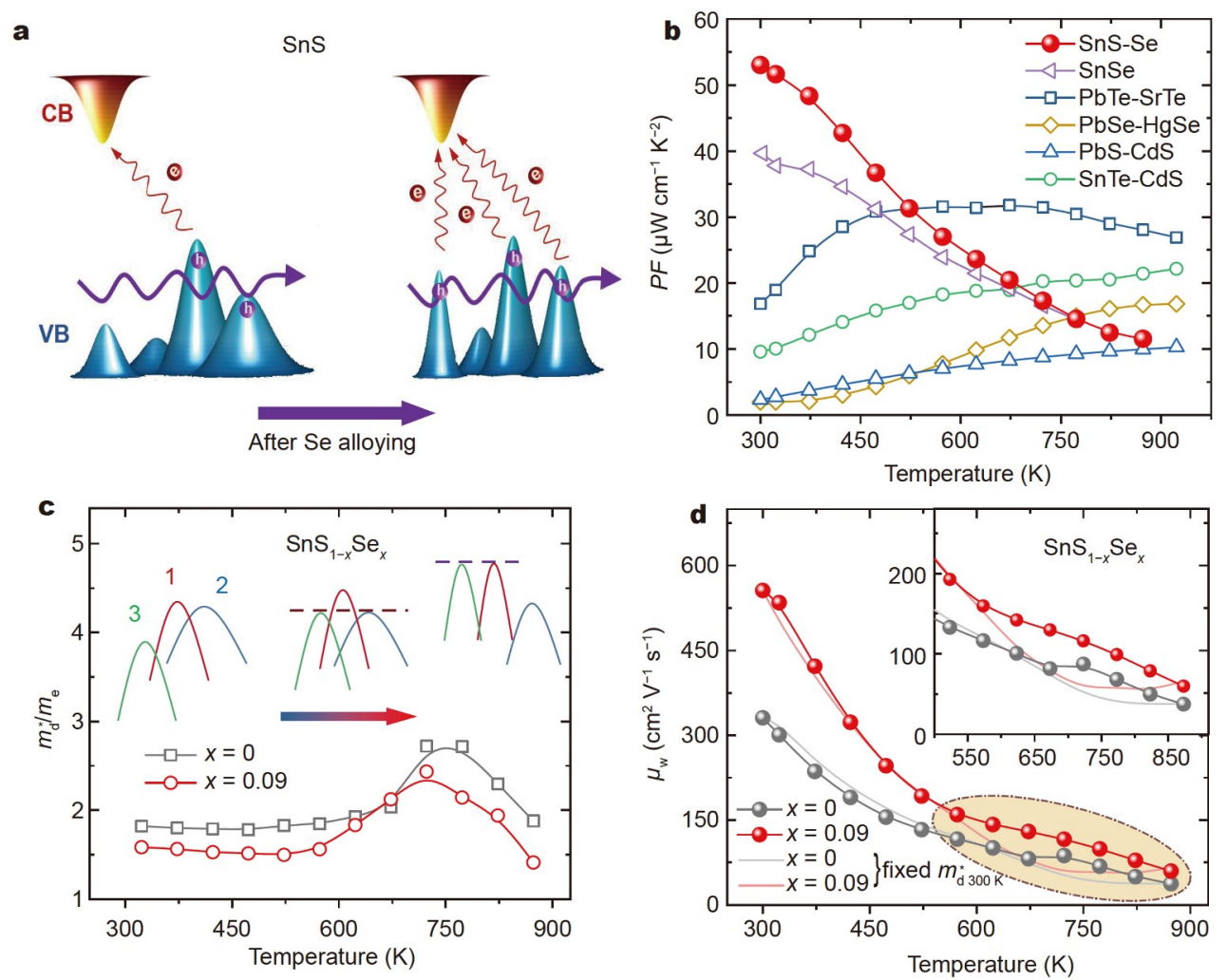

Figure 5 Electrical transport properties as a function of temperature for SnS crystals with Se alloying. (a) Schematic diagram of electronic band structures for SnS with Se alloying. (b) PF comparisons of group IV-VI compounds. Reproduced with permission from Ref. [36]. Copyright 2019, The American Association for the Advancement of Science. (c) Schematic of dynamic evolution of three separate valence bands with increasing temperature for SnS (top) and $m_{\mathrm{d}}^{*} / m_{\mathrm{e}}$. (d) Weighted mobility.

alloying, the valence bands in SnS will be sharping and the energy offset between these bands can be adjusted, as shown in Fig. 5a. Consequently, a high carrier mobility is achieved, thus promoting the electrical conductivity. Besides, the high Seebeck coefficient can be maintained due to the multiple bands and properly adjusted energy difference between them. Hence, Se alloying brings about a synergetic optimization between $\mu$ and $m^{*}$, the $P F$ enhances from $\sim 30$ to $\sim 50 \mu \mathrm{W} \mathrm{cm}{ }^{-1} \mathrm{~K}^{-2}$ at room temperature, even higher than that of the state-of-art SnSe [72]. When compared with those of other thermoelectric materials in the group IV-VI compounds, it exhibits superiority in the lowtemperature region, as shown in Fig. 5b [31,85-87].

Certainly, the temperature dependence of lattice change (thermal expansion) can affect the electronic band structure $[88,89]$. Therefore, the temperature-dependent lattice parameters and atomic positions in SnS can be obtained from the high-temperature synchrotron radiation XRD (SR-XRD) measurements, then using DFT calculations to obtain the electronic band structures for better understanding the dynamic synergy between $\mu$ and $m^{*}$. The DFT calculation results show the three separate electronic valence band evolution behaviors with rising temperature, including two-band divergence, convergence, and crossing, as shown in Fig. 5c. Specifically, the energy offset between VBM1 and VBM2 is $\sim 0.07 \mathrm{eV}$ while that between VBM1 and VBM3 is $\sim 0.12 \mathrm{eV}$ at $323 \mathrm{~K}$. With increasing temperature, VBM1 and VBM3 move up and end up with convergence while VBM2 moves away from VBM1 (divergence); meanwhile VBM2 and VBM3 experience band crossing (convergence and divergence) at $\sim 650 \mathrm{~K}$. After Se alloying, all the three bands become sharping and the temperature-dependent triple-band evolution behavior can be promoted [34]. The electronic band structure evolution is associated with the electrical transport properties, involving the dynamic variation between $\mu$ and $m^{*}$. Herein, the weighted mobility $\left(\mu_{\mathrm{W}}\right)$ is introduced, which is a good descriptor of the $\mu$ and $m^{*}$, and can be used to estimate the degree of synergetic optimization between these two parameters [90-92]. Moreover, the $\mu_{\mathrm{w}}$ can be directly extracted from the measured Seebeck coefficient and electrical conductivity, offering a convenient way to evaluate the temperature-dependent carrier transport behaviors. Briefly, the weighted mobility $\mu_{\mathrm{w}}$ can be expressed as $\mu_{\mathrm{w}} \approx \mu_{\mathrm{H}}\left(m_{\mathrm{d}}{ }^{*} / m_{\mathrm{e}}\right)^{3 / 2}$, and hence the $m_{\mathrm{d}}{ }^{*}$ can be estimated approximatively through the weighted mobility $\mu_{\mathrm{w}}$ divided by Hall mobility $\mu_{\mathrm{H}}$ (Fig. $5 \mathrm{c}$ ). Obviously, the decline in $m_{\mathrm{d}}{ }^{*}$ confirms the band sharping after Se alloying as mentioned above. In addition, the value variation in $m_{\mathrm{d}}{ }^{*} / m_{\mathrm{e}}$ experiences three stages with rising temperature. Namely, the value is slightly changed before $\sim 600 \mathrm{~K}$, then begins to increase and reaches the peak at $\sim 750 \mathrm{~K}$, and subsequently decreases back to the initial state. This process corresponds to the band evolution behavior in SnS. The increase in $m_{\mathrm{d}}{ }^{*} / m_{\mathrm{e}}$ after $\sim 600 \mathrm{~K}$ is the evidence of band convergence between VBM2 and VBM3, and subsequent decline after $\sim 750 \mathrm{~K}$ explains the band divergency with VBM3 (lighter band) rising and VBM2 (heavier band) descending. Besides, the relevant temperature points about band convergence and divergence have been promoted after Se alloying. And the improved weighted mobility with Se alloying proves it, indicating the synergetic optimization between $\mu$ and $m^{*}$ (Fig. $5 \mathrm{~d}$ ). On the other hand, if there is no 
fluctuation of three valence bands, namely, the $m_{\mathrm{d}}{ }^{*}$ remains constant with increasing temperature, the simulated weighted mobility (fix $m_{\mathrm{d}}{ }^{*}$ at room temperature) shows a distinct deviation above $\sim 600 \mathrm{~K}$ when compared with the experimental $\mu_{\mathrm{w}}$, that is, the simulated one is inferior to the latter. This indicates that the band evolution contributes to the improvement of electrical transport, especially in the high-temperature region as it can optimize the $\mu$ and $m^{*}$. To sum up, the excellent electrical transport properties in SnS originated from the interplay of three separate electronic valence bands, and the evolution behavior can be well evaluated through the weighted mobility $\mu_{\mathrm{W}}$. Furthermore, the optimization becomes more prominent after Se alloying.

\section{Explorations of n-type SnS}

As described above, serval strategies for improving the electrical transport performance have been achieved in p-type SnS. However, the progress on the n-type is slow. The main reason is that $\mathrm{SnS}$ is an intrinsic p-type semiconductor, and realizing the n-type conversion is difficult owing to the low formation energy of Sn vacancy $[93,94]$. The calculated defect formation enthalpies $(\Delta H)$ of intrinsic defects under the S-poor and S-rich conditions are shown in Fig. 6a, including the vacancies $\left(\mathrm{V}_{\mathrm{Sn}}\right.$ and $\left.\mathrm{V}_{\mathrm{S}}\right)$, antisites $\left(\mathrm{Sn}_{\mathrm{S}}\right.$ and $\left.\mathrm{S}_{\mathrm{Sn}}\right)$ and interstitials $\left(\mathrm{Sn}_{\mathrm{i}}\right.$ and $\left.\mathrm{S}_{\mathrm{i}}\right)$. Under the $\mathrm{S}$-rich condition, $\mathrm{V}_{\mathrm{Sn}}$ has the lowest $\Delta H$ and acts as a shallow acceptor in SnS, which is mainly responsible for p-type conduction. In the $\mathrm{S}$-poor limits, the $\mathrm{V}_{\mathrm{S}}$ has a lower $\Delta H$ than $\mathrm{V}_{\mathrm{Sn}}$, but it cannot effectively compensate the holes produced by $\mathrm{V}_{\mathrm{Sn}}$ due to that the $\mathrm{V}_{\mathrm{S}}$ and $\mathrm{Sn}_{\mathrm{i}}$ transition levels are closer to the VBM rather than the conduction band minimum (CBM) [93]. Therefore, under the S-poor condition, improving the chemical environment of defects in SnS, such as generating new donor defects or further reducing the $\Delta H$ of n-type defects, may be achievable to the n-type conduction. On the other hand, the electrical transport is related to the electronic band structure in semiconductors, which is made of atomic orbitals [73]. Hence, it is helpful to understand the electrical transport nature, and analyzing the contribution of each atom can provide a constructive guideline for performance optimization in SnS [56]. The projected DOS (PDOS) near the band edges is conducted through DFT calculations, which can reflect the charge density contribution, as shown in Fig. 6b. It can be seen the total DOS in VBM (p-type) in SnS is composed of both Sn-s and S-p orbitals while the Sn-p predominately contributes to the DOS in the CBM (n-type). Specifically, the $\mathrm{Sn}$-s and S- $\mathrm{p}_{z}$ orbitals contribute to the DOS equally to the VBM, indicating a tendentious charge density contribution within the plane in p-type $\mathrm{SnS}$. This can explain why the superior electrical transport properties are obtained along the in-plane direction in p-type SnS from the view of atomic orbitals theory. On the other hand, Sn- $\mathrm{p}_{y}$ mainly contributes to the total DOS in the CBM and then $\mathrm{Sn}-\mathrm{p}_{x}$ takes the place with increasing energy, which implies that the charge density largely contributes to the in-plane direction at low concentrations and then inclines to the out-of-plane direction with rising carrier concentration in n-type SnS. Therefore, the DFT results manifest that the electrical transport properties
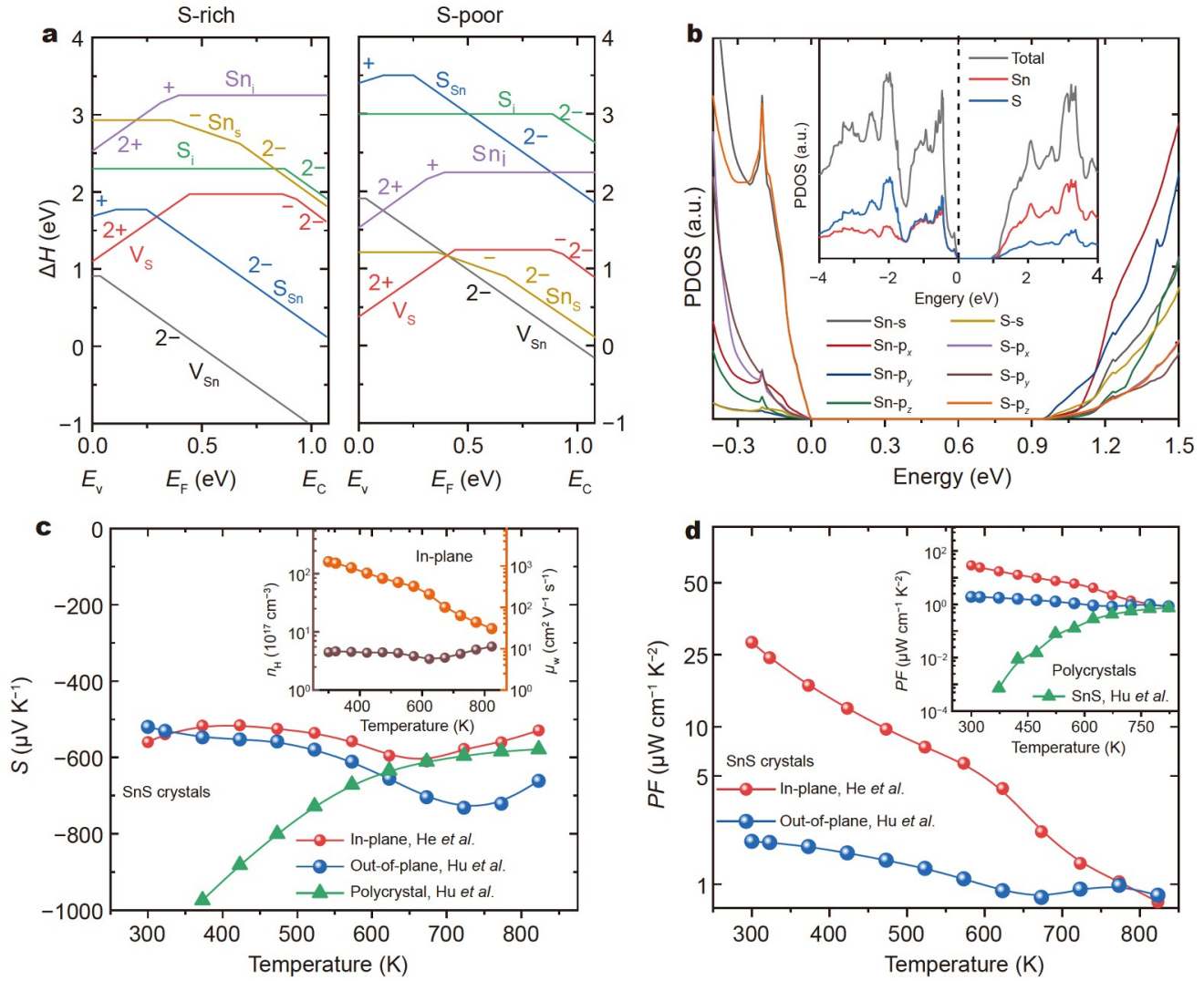

Figure 6 Calculated formation enthalpy and PDOS, and electrical transport properties as a function of temperature for n-type SnS. (a) Calculated formation enthalpies $\Delta H$ of intrinsic point defects. Reproduced with permission from Ref. [93]. Copyright 2015, AIP Publishing. (b) PDOS near the CBM and VBM. The inset shows the total DOS and the contribution of each atom. (c) Seebeck coefficient. Inset shows the carrier concentration and Hall mobility of SnS crystals. (d) PF. Reproduced with permission from Ref. [56]. Copyright 2021, Science China Press and Springer-Verlag GmbH Germany, part of Springer Nature. 
along the in-plane direction outperform the out-of-plane direction at low carrier concentrations. However, the superior out-of-plane electrical transport properties are expected once the carrier concentration is optimized in n-type $\mathrm{SnS}$.

Experimentally, two strategies are adopted to realize the ntype conduction in $\mathrm{SnS}$, including cation $\left(\mathrm{Pb}^{2+}, \mathrm{Sb}^{3+}, \mathrm{Bi}^{3+}\right)[95-$ 97] substitution in $\mathrm{Sn}$ sites and halogen anion $\left(\mathrm{Cl}^{-}, \mathrm{Br}^{-}\right)$ $[50,98,99]$ doping at $\mathrm{S}$ sites. The carrier concentration, however, is too low to achieve a good electrical conductivity. It is encouraging that the carrier mobility can be improved through crystal growth compared with the polycrystalline form, thus compensating the lack contribution of carrier concentration to electrical conductivity to some extent $[50,56,100]$. As shown in Fig. 6c, benefiting from the low carrier concentration $\left(\sim 4.5 \times 10^{17} \mathrm{~cm}^{-3}\right)$, a large Seebeck coefficient can be obtained in n-type SnS crystals. Moreover, owing to the high in-plane carrier mobility of crystals, a moderate electrical conductivity can be realized, thus giving rise to a high $P F$ of $\sim 28 \mu \mathrm{W} \mathrm{cm} \mathrm{cm}^{-1} \mathrm{~K}^{-1}$ at room temperature (Fig. $6 \mathrm{~d}$ ). It should be noted that the electrical transport properties along the in-plane direction crystals are higher than that along the out-of-plane direction in n-type SnS, which is consistent with the DFT calculation results mentioned in Fig. $6 b$.

\section{ANHARMONICITY AND LOW THERMAL CONDUCTIVITY}

For bulk materials, it is inevitable that introducing structural defects with multiple scales to reduce the thermal conductivity will paradoxically affect the carrier transport. Therefore, under the condition of ensuring high electrical transports, the extra scattering centers of phonons should be avoided as many as possible. In contrast, if a material with intrinsically low thermal conductivity is sought, the thermoelectric performance optimization can be simplified, making it an independently well-optimized parameter. The intrinsically low thermal conductivity in materials is related to the bonding of atoms, the vibration mode, and the environment around the atoms. It originates from many aspects, such as strong anharmonicity [59,101-103], anisotropic bonding $[27,104]$, lone-pair electrons $[17,105,106]$, and complex crystal structure [107-109].

Above the Debye temperature, assuming the phonon transport is dominated by an Umklapp scattering process, the lattice thermal conductivity $\kappa_{\text {lat }}$ can be expressed as [110]

$\kappa_{\text {lat }}=A \frac{M \theta_{\mathrm{D}}^{3} V}{\gamma^{2} N^{2 / 3} T}$,

where $A$ is a physical constant, $M$ is the average atomic mass, $\theta_{\mathrm{D}}$ is the Debye temperature, $V$ is the volume of each atom, $N$ is the number of atoms in a unit cell, and $\gamma$ is the Grüneisen parameter. Generally, the strength of anharmonicity in a material can be expressed by $\gamma$, the stronger interaction between phonons, the larger $\gamma$, thus the lower $\kappa_{\text {lat }}$. Owing to the layered structure with low symmetry and anisotropic bonding, SnS exhibits strong anharmonicity. As shown in Fig. 7a, the calculated Grüneisen parameters in SnS are 4.03, 1.92 and 2.96 along $a, b$ and $c$ axis, respectively, showing a strong anisotropic feature along three crystallographic directions. In addition, the calculated theoretical minimum lattice thermal conductivities are $\kappa_{\min }{ }^{a}=$ $0.35 \mathrm{~W} \mathrm{~m}^{-1} \mathrm{~K}^{-1}, \kappa_{\min }{ }^{b}=0.45 \mathrm{~W} \mathrm{~m}^{-1} \mathrm{~K}^{-1}, \kappa_{\min }{ }^{c}=0.52 \mathrm{~W} \mathrm{~m}^{-1} \mathrm{~K}^{-1}$, respectively [34]. The corresponding $\kappa_{\text {lat }}$ in $\mathrm{SnS}$ crystals along the
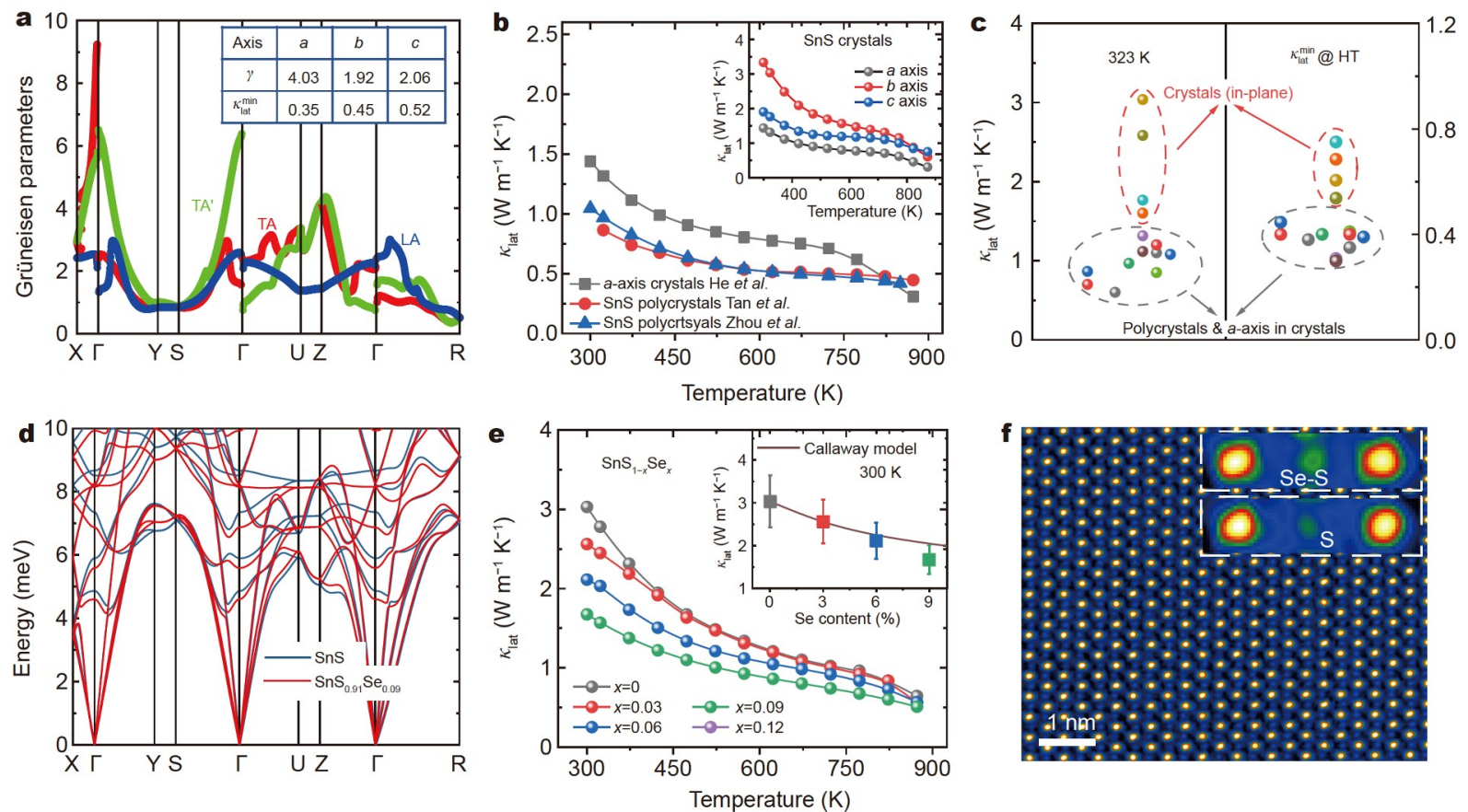

Figure 7 Phonon dispersions and thermal conductivity for SnS. (a) Grüneisen parameters. Reproduced with permission from Ref. [34]. Copyright 2018, the Royal Society of Chemistry. (b) Comparisons in lattice thermal conductivity between SnS crystals and polycrystalline samples. (c) Lattice thermal conductivities at $323 \mathrm{~K}$ and high temperature limit for SnS-based samples. (d) Phonon band structures of SnS with Se alloying. Reproduced with permission from Ref. [36]. Copyright 2019, The American Association for the Advancement of Science. (e) Lattice thermal conductivity after Se alloying. Inset shows the lattice thermal conductivities fitted with the Callaway model at room temperature. (f) Atomically resolved STEM-high angle annular dark field (HAADF) image confirms the Se substitution to S sites. Reproduced with permission from Ref. [36]. Copyright 2019, The American Association for the Advancement of Science. 
three axes directions are depicted in the inset of Fig. 7b. The $\gamma$ along the $a$-axis is larger than those along both $b$ and $c$ directions, which gives rise to a distinctly low $\kappa_{\text {lat }}$ along the $a$-axis (Fig. 7c). For polycrystalline samples, however, the lattice thermal conductivities are even lower than that along $a$-axis in $\mathrm{SnS}$ crystals, due to the extra grain boundary scattering and increased scattering centers caused by defects in polycrystals. The $\kappa_{\text {lat }}$ in the crystals range from $\sim 1.5$ to $3.0 \mathrm{~W} \mathrm{~m}^{-1} \mathrm{~K}^{-1}$ at $323 \mathrm{~K}$ and decrease to the minimum of $\sim 0.5 \mathrm{~W} \mathrm{~m}^{-1} \mathrm{~K}^{-1}$ at high temperatures along the in-plane directions ( $b$ or $c$-axis), while the values in polycrystalline samples or the $a$-axis are below $\sim 1.5 \mathrm{~W} \mathrm{~m}^{-1} \mathrm{~K}^{-1}$ at $323 \mathrm{~K}$ and decline to $\sim 0.4 \mathrm{~W} \mathrm{~m}^{-1} \mathrm{~K}^{-1}$ at high temperatures. Hence, the lower $\kappa_{\text {lat }}$ is usually obtained in the polycrystals, and closer to the theoretical limit. Recently, Zhou et al. [67] have reported an ultralow lattice thermal conductivity in polycrystalline $\mathrm{SnSe}$ by purifying the reagents and removing the tin oxides covered on the surface of powders. This indicates that the tin oxides could also exist in the polycrystalline SnS during the synthesis and sintering process, and the net lattice thermal conductivity would be much lower than the results reported in the literatures presently.

In general, the lattice thermal conductivity is predominated by the acoustic phonon contribution due to the higher frequency in optical phonon [55]. However, if the frequency of optical branch is low and comparable to the acoustic branch in a material, it could contribute equally to the lattice thermal conductivity and even lead to a lower $\kappa_{\text {lat }}$. As shown in Fig. $7 d$, after Se alloying, the optical branches reveal a softening trend in the whole Brillouin zoon compared with the calculated phonon dispersions in SnS. Further, the softened optical branches are coupled with acoustic branches, and this interaction between optical and acoustic modes gives rise to a lower $\kappa_{\text {lat }}$ after Se alloying [36]. With increasing Se content, the $\kappa_{\text {lat }}$ decreases from $\sim 3.0$ to $\sim 1.7 \mathrm{~W} \mathrm{~m}^{-1} \mathrm{~K}^{-1}$ at room temperature, which agrees with the Callaway model prediction (Fig. 7e). Besides, the Se substitution in $\mathrm{SnS}$ can be observed by aberration corrected scanning transmission electron microscopy (STEM). According to the $Z$ contrast image $\left(Z_{\mathrm{Se}}=34, Z_{\mathrm{S}}=16\right)$, the abnormally brighter contrast on $S$ sites proves the substitution of Se (Fig. 7f).

\section{ANALYSIS OF QUALITY FACTOR AND ZT VALUE}

The research on the thermoelectric materials is aimed at screening out good thermoelectrics and achieving the possible maximum $Z T$ through further performance optimization. However, $Z T$ measurements for the samples do not directly offer any message of the potential property improvement in materials, namely, the initially measured $Z T$ cannot predict the finally optimal $Z T$ after carrier concentration tuning. Therefore, it is essential to predict the optimum doping level for a given material to realize its maximum $Z T$.

The quality factor analysis is based on an effective mass model that considers $Z T$ as a function of two independent variables: the reduced Fermi level $\eta$ and quality factor $B$ [111]. The $\eta$ is a function of doping and temperature, namely, $\eta=E_{\mathrm{F}} / k_{\mathrm{B}} T$, which can be derived from Seebeck coefficient. The $B$ factor is an inherent feature of material, and independent of doping level, which can be express as [111]

$B=\left(\frac{k_{\mathrm{B}}}{e}\right)^{2} \frac{\sigma_{\mathrm{E}}}{\kappa_{\text {lat }}} T$

where $\sigma_{\mathrm{E}}$ is a transport coefficient that can describe the con- ductive "quality" in the material at a given $\eta$, which is also a function of weighted mobility $\mu_{\mathrm{w}}$ mentioned in the above section [91]:

$\sigma_{\mathrm{E}}=\frac{8 \pi e\left(2 m_{\mathrm{e}} k_{\mathrm{B}} T\right)^{3 / 2}}{3 h^{3}} \mu_{\mathrm{W}}$.

In addition, $\sigma_{\mathrm{E}}$ can be estimated from both Seebeck coefficient $S$ and electrical conductivity $\sigma$. Thus, $\sigma_{\mathrm{E}}$ can also be evaluated by

$\sigma_{\mathrm{E}}=\sigma \times \exp \left(\frac{|S|}{k_{\mathrm{B}} / e}-2\right)$.

On the other hand, the $\sigma$ as a function of $\sigma_{\mathrm{E}}$ can be written as $\sigma=\sigma_{\mathrm{E}} \times \ln \left(1+e^{\eta}\right)$.

Here, the $\sigma$ also depends on the $\eta$, which describes the variation in the carrier concentration (doping level) of a material and reflects the change in the Fermi level. From Equations (5) and (6), the Seebeck coefficient $|S|$ can be considered as a function of only $\eta$, and $|S(\eta)|$ is an indicator of Fermi level. Therefore, to obtain the optimum ZT in a material, the corresponding $|S|$ should be adjusted to an optimal value through tuning the carrier concentration. Moreover, the Lorenz number $L$ also depends on the $\eta$, which can be approximately expressed using the $|S|$ :

$L\left(10^{-8} \mathrm{~W} \Omega \mathrm{K}^{-2}\right) \approx 1.5+\exp \left(-\frac{|S|}{116\left(\mu \mathrm{V} \mathrm{K}^{-1}\right)}\right)$.

Therefore, the $Z T$ can be separated to $B$ and the $\eta$-dependent terms, written as

$$
\begin{aligned}
Z T & =\frac{S^{2} \sigma T}{\kappa_{\text {lat }}+\kappa_{\text {ele }}}=\frac{S^{2}}{\frac{\kappa_{\text {lat }}}{\sigma T}+L}=\frac{S^{2}(\eta)}{\frac{\kappa_{\text {lat }}}{\sigma_{\mathrm{E}} \ln \left(1+e^{\eta}\right) T}+L(\eta)} \\
= & \frac{S^{2}(\eta)}{\frac{\left(k_{\mathrm{B}} / e\right)^{2}}{B \ln \left(1+e^{\eta}\right)}+L(\eta)},
\end{aligned}
$$

where $B$ is the defined dimensionless quality factor in Equation (3), which contains all $\eta$-independent parameters of materials. The ZT curves depending on these two parameters are plotted, as shown in Fig. 8a.

At a given quality factor $B$, the maximum $Z T\left(Z T_{\max }\right)$ can be achieved through tuning the carrier concentration $(\eta)$. Besides, under different $B$ factors, the curve of $Z T_{\max } v s . B$ factor can be derived by extracting the $Z T_{\max }$ at optimal $\eta$. As shown in Fig. $8 \mathrm{~b}$, the lager $B$, the higher optimum $Z T_{\max }$. The corresponding Seebeck coefficient at optimal $\eta$ is also obtained under different $B$ factors. Therefore, to attain $Z T_{\max }$ at a given $B$, the optimization on $\eta$ can also be realized towards the Seebeck coefficient. In p-type SnS-based samples, the $B$ factor can be enhanced through crystal growth (improving $\mu$ ) and Se alloying (synergetic optimization between $\mu$ and $m^{*}$ ). At room temperature, the $B$ factor is $\sim 0.06$ in SnS crystals and increases to $\sim 0.23$ after Se alloying. This indicates that reasonable design and utilization in the inherent features of a material is an effective way to promote the $B$ factor. It can also be seen that, the calculated quality factor $B$ from Equations (3) and (4) with corresponding $Z T$ values in $S n S$-based samples falls on the $Z T_{\max } v s . B$ curve at room temperature, indicating that the carrier concentration and Seebeck coefficient are well optimized. However, under the calculated $B$, the experimental $Z T$ values are deviated from the $Z T_{\max }$ curve at high temperatures, implying a further 

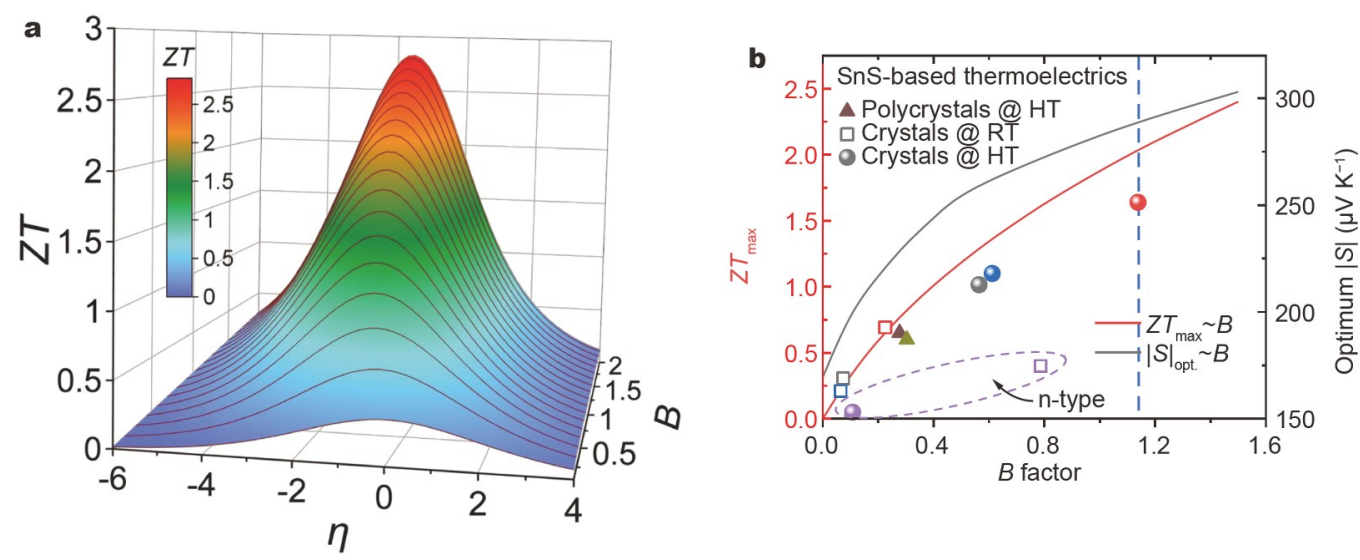

Figure $8 Z T$ values as a function of the reduced Fermi level $\eta$ and quality factor $B$. (a) $Z T$ values as a function of $\eta$ and $B$. (b) The maximum $Z T$ at optimum $\eta$ as a function of $B$ (red line). Optimum Seebeck coefficients are also plotted as a guide for optimization (black line).

room for ZT optimization. For example, the quality factor $B$ in SnS crystals with $9 \%$ Se alloying is $\sim 1.64$ at $873 \mathrm{~K}$; if the Seebeck coefficient can be optimized to $\sim 290 \mu \mathrm{V} \mathrm{K}^{-1}$ via tuning the carrier concentration, the $Z T$ can excess $\sim 2.0$. Apart from the $\mathrm{p}$ type, there is an expectation in improving $Z T$ for n-type SnSbased thermoelectrics through either enhancing $B$ factor by means of utilizing the material "nature" or further tuning carrier concentration at optimum $B$ factor.

\section{SUMMARY AND OUTLOOK}

In this work, we have reviewed and made a summary on the new emerged SnS-based thermoelectric system, including the anisotropic crystal structure, remarkable electronic band structure and synergetic interaction between valence bands, anharmonicity and the phonon "softening" behavior, and establishment of maximum $Z T$ and quality factor $B$ for performance prediction. As a simple binary compound, SnS has been reported as very promising thermoelectrics and received ever-increasing attention since 2014. The intrinsically low thermal conductivity produced by strong anharmonicity in SnS simplifies the optimization strategy to enhance $Z T$, so we only need to put focus on improving the electrical transport properties. Nowadays, many effective approaches have been implemented successfully in this system. To increase the electrical conductivity, the carrier concentration tuning and mobility promotion can be realized simultaneously through effective doping and high in-plane mobility utilization of its crystal form, respectively. Additionally, carrier concentration increment can activate the multi-valenceband transport to produce large Seebeck coefficient and high $P F$, which arises from the complex electronic band structure induced by the asymmetric crystal structure. Further, the synergetic optimization between carrier concentration and mobility through band manipulation promotes higher PFs. Consequently, these features in $\mathrm{SnS}$ allow a wide temperature plateau for high $Z T$ s, which is crucial to an overall thermoelectric conversion efficiency. Certainly, from the view of formation energy, p-type $\mathrm{SnS}$ is easier to obtain and optimize than the n-type, but some breakthroughs have also been made as to the latter. Moreover, the maximum $Z T$ values are predicted for both p- and n-type SnS systems, implying an exception for further performance enhancement.

Although numerous efforts on thermoelectric SnS have been devoted in past few years, research on this promising material is still at a relatively infant stage. Hence, some fundamental and technical issues need to be investigated for further performance improvement as predicted above. (1) Recent studies reveal that the tin oxides are readily produced during the synthesis of polycrystalline $\mathrm{SnSe}$ samples, which results in an overestimation of thermal conductivity [67]. This phenomenon may also exist in SnS. Therefore, removing the tin oxides with high thermal conductivity and purifying the polycrystalline samples would further reduce the thermal conductivity in $\mathrm{SnS}$, and thus an enhancement of final ZTs can be expected. (2) The thermal conductivity in polycrystalline samples is lower than in the crystals, but the electrical transport properties are obviously inferior to the latter due to low carrier mobility caused by grain boundary. Increasing the grain size of SnS properly, texturing or modulation doping can improve the carrier mobility and thus electrical conductivity, and possibly a high thermoelectric performance can be achieved under the condition of ensuring low thermal conductivity. (3) For SnS crystals, the PFs at high temperatures can be further promoted as predicted through introducing temperature-dependence dopant or phase transition restructuring (coexistence of Pnma and $\mathrm{Cmcm}$ ) to tune the carrier concentration, and a high $Z T$ of excessing $\sim 2.0$ is anticipated. (4) Although increasing progress on p-type $\mathrm{SnS}$ has been made and high $Z T$ values over $\sim 1.0$ have been achieved, it is urgent to obtain an n-type counterpart to match the p-type performance. Hence, extensive investigations are warranted for n-type SnS through changing the formation enthalpy of defects (such as Sn vacancy) or improving the overall chemical environment of this compound via alloying with $\mathrm{Pb}$ on $\mathrm{Sn}$ sites. Therefore, developing high-performance SnS-based thermoelectrics for both $\mathrm{p}$ - and n-type is expectant for promoting the practical applications of this low-cost thermoelectric device.

Received 10 December 2021; accepted 22 December 2021; published online 11 February 2022

1 Tan G, Zhao LD, Kanatzidis MG. Rationally designing high-performance bulk thermoelectric materials. Chem Rev, 2016, 116: 12123 12149

2 Zhang X, Zhao LD. Thermoelectric materials: Energy conversion between heat and electricity. J Materiomics, 2015, 1: 92-105

3 Qin B, Wang D, Zhao LD. Slowing down the heat in thermoelectrics. InfoMat, 2021, 3: 755-789

4 Shi XL, Zou J, Chen ZG. Advanced thermoelectric design: From materials and structures to devices. Chem Rev, 2020, 120: 7399-7515 
5 Xiao Y, Zhao LD. Charge and phonon transport in PbTe-based thermoelectric materials. npj Quant Mater, 2018, 3: 55

6 Chang C, Tan G, He J, et al. The thermoelectric properties of SnSe continue to surprise: Extraordinary electron and phonon transport. Chem Mater, 2018, 30: 7355-7367

7 He J, Tritt TM. Advances in thermoelectric materials research: Looking back and moving forward. Science, 2017, 357:

8 Qian X, Wu H, Wang D, et al. Synergistically optimizing interdependent thermoelectric parameters of n-type PbSe through alloying CdSe. Energy Environ Sci, 2019, 12: 1969-1978

9 Qin BC, Xiao Y, Zhou YM, et al. Thermoelectric transport properties of Pb-Sn-Te-Se system. Rare Met, 2017, 37: 343-350

$10 \mathrm{Qu} \mathrm{WW}$, Zhang XX, Yuan BF, et al. Homologous layered $\mathrm{InFeO}_{3}(\mathrm{ZnO})_{m}$ : New promising abradable seal coating materials. Rare Met, 2018, 37: 79-94

11 Wu D, Pei Y, Wang Z, et al. Significantly enhanced thermoelectric performance in n-type heterogeneous BiAgSeS composites. Adv Funct Mater, 2014, 24: 7763-7771

12 Xiao Y, Zhao LD. Seeking new, highly effective thermoelectrics. Science, 2020, 367: 1196-1197

13 Wang D, Huang Z, Zhang Y, et al. Extremely low thermal conductivity from bismuth selenohalides with 1D soft crystal structure. Sci China Mater, 2020, 63: 1759-1768

14 Zhou Y, Zhao LD. Promising thermoelectric bulk materials with 2D structures. Adv Mater, 2017, 29: 1702676

15 Xi L, Pan S, Li X, et al. Discovery of high-performance thermoelectric chalcogenides through reliable high-throughput material screening. J Am Chem Soc, 2018, 140: 10785-10793

16 Pei Y, Wang H, Snyder GJ. Band engineering of thermoelectric materials. Adv Mater, 2012, 24: 6125-6135

17 Nielsen MD, Ozolins V, Heremans JP. Lone pair electrons minimize lattice thermal conductivity. Energy Environ Sci, 2013, 6: 570-578

18 Liu W, Tan X, Yin K, et al. Convergence of conduction bands as a means of enhancing thermoelectric performance of n-type $\mathrm{Mg}_{2} \mathrm{Si}_{1-x} \mathrm{Sn}_{x}$ solid solutions. Phys Rev Lett, 2012, 108: 166601

19 Chang C, Zhao LD. Anharmoncity and low thermal conductivity in thermoelectrics. Mater Today Phys, 2018, 4: 50-57

20 Dresselhaus $M$, Chen G, Tang M, et al. New directions for lowdimensional thermoelectric materials. Adv Mater, 2007, 19: 10431053

21 Qiu Y, Jin Y, Wang D, et al. Realizing high thermoelectric performance in GeTe through decreasing the phase transition temperature via entropy engineering. J Mater Chem A, 2019, 7: 26393-26401

22 Zhu T, Liu Y, Fu C, et al. Compromise and synergy in high-efficiency thermoelectric materials. Adv Mater, 2017, 29: 1605884

23 Liu W, Hu J, Zhang S, et al. New trends, strategies and opportunities in thermoelectric materials: A perspective. Mater Today Phys, 2017, 1: 50-60

24 Biswas K, He J, Blum ID, et al. High-performance bulk thermoelectrics with all-scale hierarchical architectures. Nature, 2012, 489: 414-418

25 Cao T, Shi XL, Zou J, et al. Advances in conducting polymer-based thermoelectric materials and devices. Microstructures, 2021, doi: 10.20517/microstructures.2021.06

26 Poudel B, Hao Q, Ma Y, et al. High-thermoelectric performance of nanostructured bismuth antimony telluride bulk alloys. Science, 2008 , 320: 634-638

27 Yan X, Poudel B, Ma Y, et al. Experimental studies on anisotropic thermoelectric properties and structures of n-type $\mathrm{Bi}_{2} \mathrm{Te}_{2.7} \mathrm{Se}_{0.3}$. Nano Lett, 2010, 10: 3373-3378

$28 \mathrm{Hu} \mathrm{L}$, Wu $\mathrm{H}$, Zhu $\mathrm{T}$, et al. Tuning multiscale microstructures to enhance thermoelectric performance of n-type bismuth-telluridebased solid solutions. Adv Energy Mater, 2015, 5: 1500411

29 Harman TC, Taylor PJ, Walsh MP, et al. Quantum dot superlattice thermoelectric materials and devices. Science, 2002, 297: 2229-2232

30 Hsu KF, Loo S, Guo F, et al. Cubic $\mathrm{AgPb}_{m} \mathrm{SbTe}_{2+m}$ : Bulk thermoelectric materials with high figure of merit. Science, 2004, 303: 818821

31 Tan G, Shi F, Hao S, et al. Non-equilibrium processing leads to record high thermoelectric figure of merit in PbTe-SrTe. Nat Commun, 2016, 7: 12167

32 Xiao $\mathrm{Y}, \mathrm{Wu} \mathrm{H}, \mathrm{Li} \mathrm{W}$, et al. Remarkable roles of $\mathrm{Cu}$ to synergistically optimize phonon and carrier transport in n-type $\mathrm{PbTe}_{\mathrm{Cu}} \mathrm{Te}$. J Am Chem Soc, 2017, 139: 18732-18738

33 Xiao Y, Wang D, Qin B, et al. Approaching topological insulating states leads to high thermoelectric performance in n-type $\mathrm{PbTe}$. J Am Chem Soc, 2018, 140: 13097-13102

34 He W, Wang D, Dong JF, et al. Remarkable electron and phonon band structures lead to a high thermoelectric performance $Z T>1$ in earthabundant and eco-friendly SnS crystals. J Mater Chem A, 2018, 6: 10048-10056

35 Wu H, Lu X, Wang G, et al. Sodium-doped tin sulfide single crystal: A nontoxic earth-abundant material with high thermoelectric performance. Adv Energy Mater, 2018, 8: 1800087

$36 \mathrm{He} \mathrm{W}$, Wang $\mathrm{D}, \mathrm{Wu} \mathrm{H}$, et al. High thermoelectric performance in low-cost $\mathrm{SnS}_{0.91} \mathrm{Se}_{0.09}$ crystals. Science, 2019, 365: 1418-1424

37 Tan Q, Zhao LD, Li JF, et al. Thermoelectrics with earth abundant elements: Low thermal conductivity and high thermopower in doped SnS. J Mater Chem A, 2014, 2: 17302-17306

38 Albers W, Haas C, Vink HJ, et al. Investigations on SnS. J Appl Phys, 1961, 32: 2220-2225

39 Zhou B, Li S, Li W, et al. Thermoelectric properties of SnS with Nadoping. ACS Appl Mater Interfaces, 2017, 9: 34033-34041

40 Chandrasekhar HR, Humphreys RG, Zwick U, et al. Infrared and Raman spectra of the IV-VI compounds SnS and SnSe. Phys Rev B, 1977, 15: 2177-2183

41 Tan Q, Li JF. Thermoelectric properties of Sn-S bulk materials prepared by mechanical alloying and spark plasma sintering. J Elec Materi, 2014, 43: 2435-2439

42 Tan Q, Wu CF, Sun W, et al. Solvothermally synthesized SnS nanorods with high carrier mobility leading to thermoelectric enhancement. RSC Adv, 2016, 6: 43985-43988

43 Jin Z, Mao T, Qiu P, et al. Thermoelectric properties and service stability of Ag-containing $\mathrm{Cu}_{2} \mathrm{Se}$. Mater Today Phys, 2021, 21: 100550

44 Tang H, Dong JF, Sun FH, et al. Adjusting Na doping via wet-chemical synthesis to enhance thermoelectric properties of polycrystalline SnS. Sci China Mater, 2019, 62: 1005-1012

45 Wang Z, Wang D, Qiu Y, et al. Realizing high thermoelectric performance of polycrystalline SnS through optimizing carrier concentration and modifying band structure. J Alloys Compd, 2019, 789: 485-492

46 Yang $\mathrm{HQ}$, Wang $\mathrm{XY}, \mathrm{Wu} \mathrm{H}$, et al. Sn vacancy engineering for enhancing the thermoelectric performance of two-dimensional SnS. J Mater Chem C, 2019, 7: 3351-3359

47 Asfandiyar, Cai B, Zhao LD, et al. High thermoelectric figure of merit $Z T>1$ in SnS polycrystals. J Materiomics, 2020, 6: 77-85

48 Niu Y, Chen Y, Jiang J, et al. Enhanced thermoelectric performance in Li doped SnS via carrier concentration optimization. IOP Conf Ser: Mater Sci Eng, 2020, 738: 012016

49 Prado-Gonjal J, Gainza J, Aguayo I, et al. High thermoelectric performance of rapidly microwave-synthesized $\mathrm{Sn}_{1-\delta} \mathrm{S}$. Mater Adv, 2020 1: $845-853$

$50 \mathrm{Hu} \mathrm{X}, \mathrm{He} \mathrm{W}$, Wang D, et al. Thermoelectric transport properties of $\mathrm{n}$ type tin sulfide. Scripta Mater, 2019, 170: 99-105

51 Huang ZC, Yao Y, Jun P, et al. Preparation and thermoelectric property of n-type SnS. J InOrg Mater, 2019, 34: 321-327

52 Chattopadhyay T, Pannetier J, Von Schnering HG. Neutron diffraction study of the structural phase transition in SnS and SnSe. J Phys Chem Solids, 1986, 47: 879-885

53 Parker D, Singh DJ. First principles investigations of the thermoelectric behavior of tin sulfide. J Appl Phys, 2010, 108: 083712

54 Hao S, Dravid VP, Kanatzidis MG, et al. Research update: Prediction of high figure of merit plateau in $\mathrm{SnS}$ and solid solution of $(\mathrm{Pb}, \mathrm{Sn}) \mathrm{S}$. APL Mater, 2016, 4: 104505

55 Guo R, Wang X, Kuang Y, et al. First-principles study of anisotropic thermoelectric transport properties of IV-VI semiconductor compounds SnSe and SnS. Phys Rev B, 2015, 92: 115202

56 He W, Hong T, Wang D, et al. Low carrier concentration leads to high 
in-plane thermoelectric performance in n-type SnS crystals. Sci China Mater, 2021, 64: 3051-3058

57 Ge ZH, Zhao LD, Wu D, et al. Low-cost, abundant binary sulfides as promising thermoelectric materials. Mater Today, 2016, 19: 227-239

58 Wei TR, Wu CF, Li F, et al. Low-cost and environmentally benign selenides as promising thermoelectric materials. J Materiomics, 2018, 4: $304-320$

59 Zhao LD, Lo SH, Zhang Y, et al. Ultralow thermal conductivity and high thermoelectric figure of merit in SnSe crystals. Nature, 2014, 508: 373-377

60 Peng K, Lu X, Zhan H, et al. Broad temperature plateau for high ZTs in heavily doped p-type SnSe single crystals. Energy Environ Sci, 2016, 9: 454-460

61 Zhao LD, Chang C, Tan G, et al. SnSe: A remarkable new thermoelectric material. Energy Environ Sci, 2016, 9: 3044-3060

62 Chang C, Wang D, He D, et al. Realizing high-ranged out-of-plane ZTs in n-type SnSe crystals through promoting continuous phase transition. Adv Energy Mater, 2019, 9: 1901334

63 Vidal J, Lany S, d'Avezac M, et al. Band-structure, optical properties, and defect physics of the photovoltaic semiconductor SnS. Appl Phys Lett, 2012, 100: 032104

64 May AF, Toberer ES, Saramat A, et al. Characterization and analysis of thermoelectric transport in n-type $\mathrm{Ba}_{8} \mathrm{Ga}_{16-x} \mathrm{Ge}_{30+x}$. Phys Rev B, 2009, 80: 125205

65 Slade TJ, Grovogui JA, Kuo JJ, et al. Understanding the thermally activated charge transport in $\mathrm{NaPb}_{m} \mathrm{SbQ}_{m+2}(\mathrm{Q}=\mathrm{S}, \mathrm{Se}, \mathrm{Te})$ thermoelectrics: Weak dielectric screening leads to grain boundary dominated charge carrier scattering. Energy Environ Sci, 2020, 13: 1509-1518

66 Wang Y, Qin B, Zhao LD. Understanding the electrical transports of p-type polycrystalline SnSe with effective medium theory. Appl Phys Lett, 2021, 119: 044103

67 Zhou C, Lee YK, Yu Y, et al. Polycrystalline SnSe with a thermoelectric figure of merit greater than the single crystal. Nat Mater, 2021, 20: 1378-1384

68 Wei W, Chang C, Yang T, et al. Achieving high thermoelectric figure of merit in polycrystalline SnSe via introducing Sn vacancies. J Am Chem Soc, 2018, 140: 499-505

69 Sarkar D, Ghosh T, Roychowdhury S, et al. Ferroelectric instability induced ultralow thermal conductivity and high thermoelectric performance in rhombohedral p-type GeSe crystal. J Am Chem Soc, 2020, 142: $12237-12244$

70 Wood M, Kuo JJ, Imasato K, et al. Improvement of low-temperature $Z T$ in a $\mathrm{Mg}_{3} \mathrm{Sb}_{2}-\mathrm{Mg}_{3} \mathrm{Bi}_{2}$ solid solution via $\mathrm{Mg}$-vapor annealing. $\mathrm{Adv}$ Mater, 2019, 31: 1902337

71 Cutler M, Leavy JF, Fitzpatrick RL. Electronic transport in semimetallic cerium sulfide. Phys Rev, 1964, 133: A1143-A1152

72 Zhao LD, Tan G, Hao S, et al. Ultrahigh power factor and thermoelectric performance in hole-doped single-crystal SnSe. Science, 2016, 351: $141-144$

73 Chang C, Wu M, He D, et al. 3D charge and 2D phonon transports leading to high out-of-plane $Z T$ in n-type SnSe crystals. Science, 2018, 360: $778-783$

74 Tang Y, Gibbs ZM, Agapito LA, et al. Convergence of multi-valley bands as the electronic origin of high thermoelectric performance in $\mathrm{CoSb}_{3}$ skutterudites. Nat Mater, 2015, 14: 1223-1228

75 Zhang Q, Liao B, Lan Y, et al. High thermoelectric performance by resonant dopant indium in nanostructured SnTe. Proc Natl Acad Sci USA, 2013, 110: 13261-13266

76 Heremans JP, Jovovic V, Toberer ES, et al. Enhancement of thermoelectric efficiency in PbTe by distortion of the electronic density of states. Science, 2008, 321: 554-557

77 Zhang J, Liu R, Cheng N, et al. High-performance pseudocubic thermoelectric materials from non-cubic chalcopyrite compounds. Adv Mater, 2014, 26: 3848-3853

78 Pei Y, Shi X, LaLonde A, et al. Convergence of electronic bands for high performance bulk thermoelectrics. Nature, 2011, 473: 66-69

79 Roychowdhury S, Biswas K. Slight symmetry reduction in thermoelectrics. Chem, 2018, 4: 939-942

80 Airapetyants S, Vinogradova M, Dubrovskaya I, et al. Structure of the valence band of heavily doped lead telluride. Sov Phys Solid State USSR, 1966, 8: 1069-1072

81 Wang S, Zheng G, Luo T, et al. Exploring the doping effects of Ag in p-type $\mathrm{PbSe}$ compounds with enhanced thermoelectric performance. J Phys D-Appl Phys, 2011, 44: 475304

82 Qin Y, Hong T, Qin B, et al. Contrasting $\mathrm{Cu}$ roles lead to high ranged thermoelectric performance of $\mathrm{PbS}$. Adv Funct Mater, 2021, 31: 2102185

83 Kafalas JA, Brebrick RF, Strauss AJ. Evidence that SnTe is a semiconductor. Appl Phys Lett, 1964, 4: 93-94

84 Rowe, D., Thermoelectrics Handbook: Macro to Nano CRC. Boca Raton: CRC Press, 2006

85 Tan G, Shi F, Hao S, et al. Codoping in SnTe: Enhancement of thermoelectric performance through synergy of resonance levels and band convergence. J Am Chem Soc, 2015, 137: 5100-5112

86 Zhao LD, He J, Hao S, et al. Raising the thermoelectric performance of p-type $\mathrm{PbS}$ with endotaxial nanostructuring and valence-band offset engineering using CdS and ZnS. J Am Chem Soc, 2012, 134: 1632716336

87 Hodges JM, Hao S, Grovogui JA, et al. Chemical insights into PbSe- $x \%$ HgSe: High power factor and improved thermoelectric performance by alloying with discordant atoms. J Am Chem Soc, 2018, 140: 1811518123

88 Božin ES, Malliakas CD, Souvatzis $\mathrm{P}$, et al. Entropically stabilized local dipole formation in lead chalcogenides. Science, 2010, 330: 1660-1663

89 Huang Z, Zhao LD. Symmetry and asymmetry in thermoelectrics. J Mater Chem C, 2020, 8: 12054-12061

90 He W, Qin B, Zhao LD. Predicting the potential performance in ptype $\mathrm{SnS}$ crystals via utilizing the weighted mobility and quality factor. Chin Phys Lett, 2020, 37: 087104

91 Snyder GJ, Snyder AH, Wood M, et al. Weighted mobility. Adv Mater, 2020, 32: 2001537

92 Qin B, He W, Zhao LD. Estimation of the potential performance in ptype SnSe crystals through evaluating weighted mobility and effective mass. J Materiomics, 2020, 6: 671-676

93 Xiao Z, Ran FY, Hosono H, et al. Route to n-type doping in SnS. Appl Phys Lett, 2015, 106: 152103

94 Malone BD, Gali A, Kaxiras E. First principles study of point defects in SnS. Phys Chem Chem Phys, 2014, 16: 26176-26183

95 Ran FY, Xiao Z, Toda Y, et al. n-type conversion of SnS by isovalent ion substitution: Geometrical doping as a new doping route. Sci Rep, 2015, 5: 10428

96 Dussan A, Mesa F, Gordillo G. Effect of substitution of Sn for Bi on structural and electrical transport properties of $\mathrm{SnS}$ thin films. J Mater Sci, 2010, 45: 2403-2407

97 Sinsermsuksakul P, Chakraborty R, Kim SB, et al. Antimony-doped tin(II) sulfide thin films. Chem Mater, 2012, 24: 4556-4562

98 Iguchi $\mathrm{Y}$, Inoue $\mathrm{K}$, Sugiyama $\mathrm{T}$, et al. Single-crystal growth of $\mathrm{Cl}$ doped n-type $\mathrm{SnS}$ using $\mathrm{SnCl}_{2}$ self-flux. Inorg Chem, 2018, 57: 67696772

99 Kawanishi S, Suzuki I, Ohsawa T, et al. Growth of large single crystals of n-type SnS from halogen-added Sn flux. Cryst Growth Des, 2020 20: 5931-5939

100 Yin Y, Cai J, Wang H, et al. Single-crystal growth of n-type $\mathrm{SnS}_{0.95}$ by the temperature-gradient technique. Vacuum, 2020, 182: 109789

101 Li CW, Hong J, May AF, et al. Orbitally driven giant phonon anharmonicity in SnSe. Nat Phys, 2015, 11: 1063-1069

102 Zhang X, Chang C, Zhou Y, et al. BiCuSeO thermoelectrics: An update on recent progress and perspective. Materials, 2017, 10: 198

103 Liu X, Wang D, Wu H, et al. Intrinsically low thermal conductivity in $\mathrm{BiSbSe}_{3}$ : A promising thermoelectric material with multiple conduction bands. Adv Funct Mater, 2019, 29: 1806558

104 Zhao LD, Berardan D, Pei YL, et al. $\mathrm{Bi}_{1-x} \mathrm{Sr}_{x} \mathrm{CuSeO}$ oxyselenides as promising thermoelectric materials. Appl Phys Lett, 2010, 97: 092118

105 Skoug EJ, Morelli DT. Role of lone-pair electrons in producing minimum thermal conductivity in nitrogen-group chalcogenide compounds. Phys Rev Lett, 2011, 107: 235901

106 Pei YL, Wu H, Sui J, et al. High thermoelectric performance in n-type BiAgSeS due to intrinsically low thermal conductivity. Energy Environ 


\section{Sci, 2013, 6: 1750}

107 Kurosaki K, Kosuga A, Muta $\mathrm{H}$, et al. $\mathrm{Ag}_{9} \mathrm{TlTe}_{5}$ : A high-performance thermoelectric bulk material with extremely low thermal conductivity. Appl Phys Lett, 2005, 87: 061919

108 Brown SR, Kauzlarich SM, Gascoin F, et al. $\mathrm{Yb}_{14} \mathrm{MnSb}_{11}$ : New high efficiency thermoelectric material for power generation. Chem Mater, 2006, 18: 1873-1877

109 Jana MK, Pal K, Warankar A, et al. Intrinsic rattler-induced low thermal conductivity in zintl type TlInTe 2 . J Am Chem Soc, 2017, 139: 4350-4353

110 Morelli DT, Jovovic V, Heremans JP. Intrinsically minimal thermal conductivity in cubic I-V-VI 2 semiconductors. Phys Rev Lett, 2008, 101: 035901

111 Dongmin Kang S, Jeffrey Snyder G. Charge-transport model for conducting polymers. Nat Mater, 2016, 16: 252-257

Acknowledgements This work was supported by the National Natural Science Foundation of China (51772012), the National Key Research and Development Program of China (2018YFA0702100), the 111 Project (B17002). Zhao L appreciates the support of the high performance computing (HPC) resources at Beihang University, and the National Science Fund for Distinguished Young Scholars (51925101).

Author contributions Zhao LD conceived the structure of this review. He $\mathrm{W}$ and Zhao LD participated in the analysis, drawing and writing of the entire perspective. Ang R and Zhao LD revised the draft before it was submitted, and all the authors co-edited the final version of this perspective.

Conflict of interest The authors declare that they have no conflict of interest.

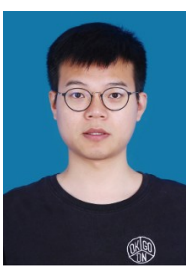

Wenke He is currently an associate research fellow at the Institute of Nuclear Science and Technology, Sichuan University. He received his BE degree from China Three Gorges University in 2016 and $\mathrm{PhD}$ degree from Beihang University in 2021. His main research interests focus on the crystal growth and performance optimization of thermoelectric materials with layered structures.

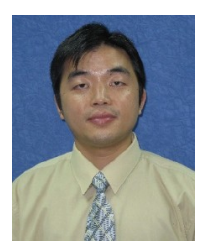

Ran Ang is a full professor at Sichuan University since 2015. He received his $\mathrm{PhD}$ degree from the Institute of Solid State Physics, Chinese Academy of Sciences, in 2008, and his postdoctoral research experience spanned 7 years during 2008-2015 from Nanyang Technological University, National University of Singapore, Tohoku University, and National Institute for Materials Science, Japan. He has been working on thermoelectric materials, superconducting materials, and magnetic materials for more than 18 years. His research interest focuses on materials physics and thermoelectric applications.

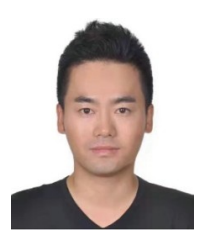

Li-Dong Zhao is a full professor of the School of Materials Science and Engineering at Beihang University, China. He received his $\mathrm{PhD}$ degree from the University of Science and Technology Beijing, China in 2009. He was a postdoctoral research associate at the Université Paris-Sud and Northwestern University from 2009 to 2014 . His research interests include electrical and thermal transport behaviors in the compounds with layered structures. Group website: http://shi.buaa.edu. cn/zhaolidong/zh_CN/index.htm.

\section{低成本SnS中卓越的电子和声子传输: 一种具有前途 的新型热电材料}

何文科 ${ }^{1,2}$, 昂然 ${ }^{1}$, 赵立东 ${ }^{2 *}$

摘要 $\mathrm{SnS}$ 作为一种看似简单的化合物, 近年来却因其极具吸引力的电 子和声子传输特性而在热电领域得到广泛关注，使其成为一种非常具 有应用前景的热电材料候选者. 本文首先讨论了 SnS的基本特性，包括 晶体结构、电子和声子能带结构以及物理和化学性质. 同时, 对提高该 材料热电性能的策略也进行了总结, 包括载流子浓度的优化和载流子 迁移率的提升, 多价带输运协同优化热电参数, 以及非简谐性和声子 “软化”行为. 对于晶体和多晶SnS在电和热传输性能上的差异, 我们也 进行了比较. 然后, 建立了预测 $S n S$ 体系中最大 $Z T$ 的理论模型计算, 以 进一步提高其热电性能. 最后, 我们提出了进一步提升 $\mathrm{SnS}$ 体系 $Z T$ 值的 方法策略. 对这种新型材料的探索和研究将有助于热电材料的实际推 广和应用, 以满足市场对低成本、高效率和环境兼容性的需求. 